Supporting Information

\title{
The Oxygen Effect in Heterobimetallic Catalysis-the Zr-O-Ti System an Excellent Example for Olefin Polymerization
}

Prabhuodeyara M.Gurubasavaraj ${ }^{\dagger}$, Herbert W. Roesky ${ }^{\dagger}$, Prabhuodeyara M. Veeresha Sharma ${ }^{\ddagger}$, Rainer B. Oswald ${ }^{\S}$, Volker Dolle ${ }^{\$}$, Regine Herbst-Irmer $^{\dagger}$, and Aritra Pal ${ }^{\dagger}$

\section{Complete Reference (22):}

Frisch, M. J.; Trucks, G. W.; Schlegel, H. B.; Scuseria, G. E.; Robb, M. A.; Cheeseman, J. R.; Montgomery, J. A.; Vreven, Jr. T.; Kudin, K. N.; Burant, J. C.; Millam, J. M.; Iyengar, S. S.; Tomasi, J.; Barone, V.; Mennucci, B.; Cossi, M.; Scalmani, G.; Rega, N.; Petersson, G. A.; Nakatsuji, H.; Hada, M.; Ehara, M.; Toyota, K.; Fukuda, R.; Hasegawa, J.; Ishida, M.;

Nakajima, T.; Honda, Y.; Kitao, O.; Nakai, H.; Klene, M.; Li, X.; Knox, J. E.; Hratchian, H. P.; Cross, J. B.; Bakken, V.; Adamo, C.; Jaramillo, J.; Gomperts, R.; Stratmann, R. E.;

Yazyev, O.; Austin, A. J.; Cammi, R.; Pomelli, C.; Ochterski, J. W.; Ayala, P. Y.; Morokuma, K.; Voth, G. A.; Salvador, P.; Dannenberg, J. J.; Zakrzewski, V. G.; Dapprich, S.; Daniels, A. D.; Strain, M. C.; Farkas, O.; Malick, D. K.; Rabuck, A. D.; Raghavachari, K.; Foresman, J. B.; Ortiz, J. V.; Cui, Q.; Baboul, A. G.; Clifford, S.; Cioslowski, J.; Stefanov, B. B.; Liu, G.; Liashenko, A.; Piskorz, P.; Komaromi, I.; Martin, R. L.; Fox, D. J.; Keith, T.; Al-Laham, M. A.; Peng, C. Y.; Nanayakkara, A.; Challacombe, M.; Gill, P. M. W.; Johnson, B.; Chen, W.; Wong, M. W.; Gonzalez, C.; Pople, J. A. Gaussian 03, Revision C.02, Gaussian, Inc., Wallingford CT, 2004. 


\section{Computational Results.}

\section{Theoretical study results on complex 1}

Table S1. Important Geometry Parameters for Complex $1\left(\mathrm{Cp}^{*} \mathrm{ZrMe}(\mathrm{OH})\right)$

\begin{tabular}{|l|l|}
\hline & \multicolumn{1}{|c|}{$\left[\boldsymbol{A}, \hat{A}^{\circ}\right]$} \\
\hline Zr-CH3 & 2.29859 \\
\hline Zr-OH & 2.02099 \\
\hline O-H & 0.96156 \\
\hline angle C-Zr-O & 93.92 \\
\hline angle H-O-Zr & 123.98 \\
\hline angle cp*-Zr-cp* & 136.96 \\
\hline dihedral angle C-Zr-O-H & 80.47409 \\
\hline
\end{tabular}

Table S2. Important Geometry Parameters for the Hydride Analogue of Complex 1 $\left(\mathrm{Cp}{ }_{2} \mathrm{ZrH}(\mathrm{OH})\right)$

\begin{tabular}{|l|l|}
\hline & \multicolumn{1}{|c|}{$\left[\boldsymbol{A}, \hat{A}^{\circ}\right]$} \\
\hline Zr-H & 1.84655 \\
\hline Zr-OH & 2.01837 \\
\hline O-H & 0.96122 \\
\hline angle H-Zr-O & 97.87 \\
\hline angle H-O-Zr & 125.58 \\
\hline angle cp*-Zr-cp* & 134.32 \\
\hline dihedral angle H-Zr-O-H & 83.57 \\
\hline
\end{tabular}




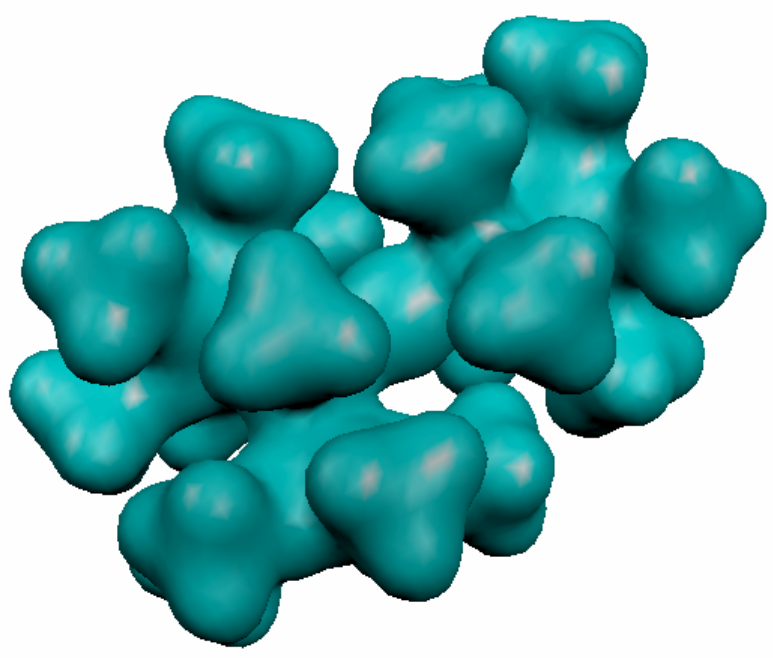

Figure S1. Space filling model of complex 2 obtained from ab initio calculation. 